\title{
Microvascular studies in human radiation bowel disease
}

\author{
N D CARR, B R PULLEN, P S HASLETON, AND P F SCHOFIELD \\ From the Department of Surgery, Christie Hospital, Manchester, Department of Pathology, Withington \\ Hospital, Manchester, and Department of Medical Biophysics, University of Manchester, Manchester
}

SUMmaRY The microvasculature was investigated in the normal bowel ( $n=43$ patients) and in radiation bowel disease $(n=18$ patients). Tissue samples obtained from postoperative colectomy specimens in which the intramural vessels had been perfused with barium sulphate suspension were examined. Microradiography was used to study vascular pattern which was abnormal in radiation bowel disease. A recently described radiograph fluorescence system was used to estimate barium concentration, and hence microvascular volume. The radiation group showed a highly significant reduction in barium concentration $(p<0 \cdot 001)$, when compared with the normal group. This reduction was diffuse in samples from 15 patients who had received combined intracavity and external radiotherapy, but localised in two patients who had received intracavity treatment only. It is concluded that microvascular compromise is an important factor in the natural history of radiation bowel disease.

Pelvic irradiation used in the treatment of uterine and bladder malignancy may produce injury to the bowel. This can occur immediately as a self limiting proctosigmoiditis. Alternatively, it may be delayed for many months or years after treatment and present as intestinal perforation, necrosis, or stricture.'

The pathological response of the bowel to radiation is complex, but occlusive changes in the small intramural vessels are commonly found at the site of radiation bowel disease. ${ }^{2}$ It has been suggested that these vascular lesions may obliterate the microvasculature of the bowel and lead to progressive ischaemia. ${ }^{34}$ Although there is evidence from microvascular studies in experimental animals to support this hypothesis, ${ }^{56}$ the role of small vessel disease in the pathogenesis of human radiation bowel disease remains unclear.

The purpose of the present study was to examine changes in the microvasculature in human radiation bowel disease.

\section{Methods \\ PATIENTS \\ CLINICAL DATA \\ Eighteen female patients who underwent resection \\ Address for correspondence: Dr N D Carr. Teaching Unit Five. Withington Hospital. Nell Lance. Manchester 20. \\ Received for publication 22 July 198.3}

for radiation bowel disease between March 1982 and March 1983 were included in the study. The age range was $26-65$ years (mean age $44 \cdot 1$ years). The initial diagnosis was carcinoma of the cervix in 17 patients and metastatic breast carcinoma in one patient. Fifteen of these patients received both external and intracavity radiotherapy, two patients intracavity treatment only, and one patient external radiotherapy alone. The time interval between therapeutic irradiation and the onset of bowel symptoms ranged from one to 28 months (mean duration 8.4 months). The site of the bowel disease was rectosigmoid (11 cases), terminal ileum (three cases), and both rectosigmoid and terminal ileum (four cases). The types of complications encountered in the rectosigmoid included necrosis with perforation (10 cases), uncomplicated stricture (two cases), rectal ulcer (two cases), and rectovaginal fistula (one case). In the terminal ileum necrosis with perforation (five cases) and stricture (two cases) occurred.

In 43 patients who had resection for carcinoma of the colon and had not received radiotherapy, areas of histologically normal bowel were studied by the same techniques. The age range was 38-82 years (mean age 65.7 years).

MATERIA L

Fresh operative specimens were used in all cases.

SPECIMEN PERFUSION

Immediately after surgical removal, the post- 
operative specimen was perfused with a $50 \%$ weight/ volume solution of barium sulphate (Micropaque), through an arterial cannula which had been introduced into one of the mesenteric arteries. The barium sulphate was infused manually using a $60 \mathrm{ml}$ bladder syringe at a pressure of $60-140 \mathrm{mmHg}$. This was continued until contrast medium flowed freely from the cut ends of the bowel, thus achieving a steady state. The arterial cannula was then removed and the cut edges of the bowel and mesentery oversewn to prevent leakage of contrast. The specimen was then fixed in $10 \%$ formalin for 48 hours.

\section{MICROVASCULAR STUDIES \\ Qualitative}

After fixation, multiple transverse tissue blocks of the bowel wall were taken. These were obtained from each specimen at measured intervals throughout its length, so as to obtain at least five blocks from each specimen. The tissue blocks were then routinely processed. (a) Histopathology: $5 \mu \mathrm{m}$ sections were cut from each block and stained with haematoxylin and eosin and elastic van Geison. These sections were studied histopathologically so that the appearances could be compared with the microradiographic features from the same block. (b) Microradiography: a $400 \mu \mathrm{m}$ section was cut from the remainder of each block. These sections were then Sellotaped to the piece of thin card which was of the same dimensions as an Industrex MX (Kodak) radiograph film. The card was then placed in direct contact with the film. Using a cabinet radiograph system (Faxitron) a radiograph of the section was taken at $10 \mathrm{Kvp}$ with an exposure time of 7.5 minutes. After development, the microradiographs obtained were studied under appropriate magnification using a microfîche projector and standard microscope.

Quantiative fluorescent radiograph analysis

Small samples of tissue $(2 \times 2 \mathrm{~cm})$ were obtained from areas immediately adjacent to the points at which the blocks for histopathological and microradiographic examination were taken. At least five samples from each specimen were studied. Each sample was dissected into a preparation consisting of mucosa-submucosa and a preparation comprising muscularis propria in order to estimate barium concentration in the whole sample and each bowel wall layer by radiograph fluorescence.

Each sample of whole bowel, muscularis, and mucosa-submucosa was exposed to $140 \mathrm{Kev} \gamma$ rays. The induced barium fluorescence was measured at $47 \mathrm{Kev}$. The $90^{\circ}$ scatter from the sample at $140 \mathrm{Kev}$, which is a measure of tissue volume, was also measured. The ratio of the number of counts obtained at $47 \mathrm{Kev}$ to those obtained at $140 \mathrm{Kev}$ for any given sample of whole bowel wall, muscularis propria or mucosa-submucosa was used as a measure of barium concentration. The reproducibility of the measurement system was in the order of $5 \% .^{7}$

\section{Results}

\section{MICRORADIOGRAPHIC FINDINGS}

\section{Normal bowel}

In the normal small and large bowel, vascular density was most pronounced in the mucosal and submucosal regions (Fig. 1). The large tortuous vessels in the submucosa gave rise to the smaller vessels which supplied the mucosa and muscle layer. The vessels in the muscularis propria formed a loose network of freely anastomosing vessels. In the colon, the mucosal capillaries were closely packed together and regularly arranged. In the ileum the vascular pattern of the villi was observed. This comprised a central arteriole which branched at the tip of the villus into a number of descending capillaries which formed a richly anastomosing vascular network on the surface of the villus. Uniform perfusion with contrast medium was always observed in all layers of the bowel wall and

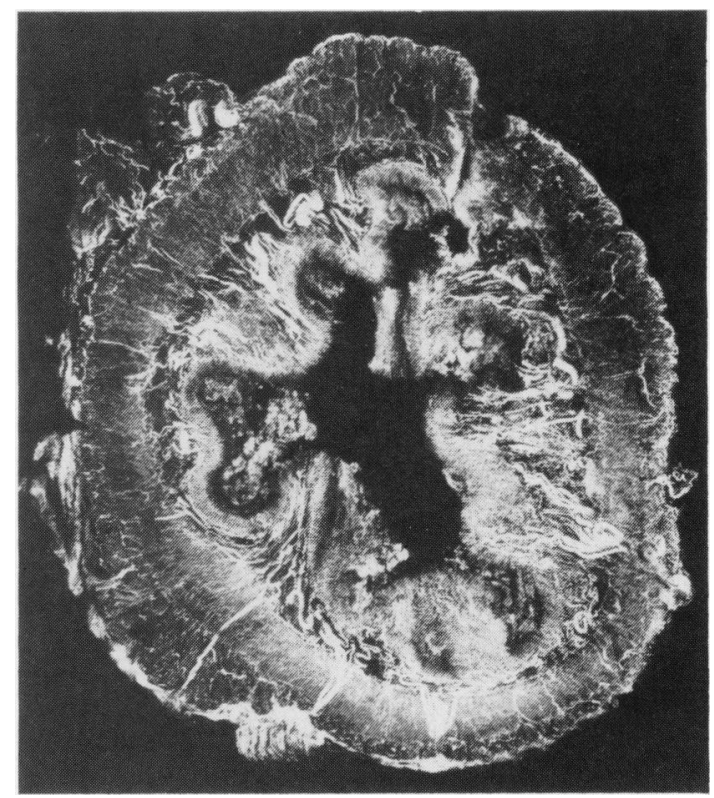

Fig. 1 Microvascular pattern in normal sigmoid colon. Dense vascular layers in mucosa and submucosa. (Orig. mag. $\times 5$ ) 
variations in luminal diameter of vessels were infrequent.

\section{Radiation bowel damage}

Alterations in microvascular architecture were present in all sections from radiation damaged bowel, but these appearances varied with the type of lesion.

At the site of fully developed strictures a reduction in vascularity affecting all layers of the intestinal wall was present. Individual vessels exhibited luminal irregularities, variations in diameter, and terminated abruptly (Fig. 2). Histologically, these areas showed pronounced fibrosis in the submucosa and muscularis propria together with severe vascular changes. These took the form of occlusive fibrin thrombi in capillaries together with severe and sometimes occlusive intimal fibrosis in the small intramural arterial vessels.

In sections taken from sites adjacent to perforations sharply demarcated areas of the bowel wall which contained no contrast medium were present (Fig. 3a). These avascular zones were either localised to part of the bowel wall or were transmural in distribution. Focal or transmural infarction in combination with fibrinoid necrosis and thrombosis of some vessels were found in these areas on histology (Fig. 3b).

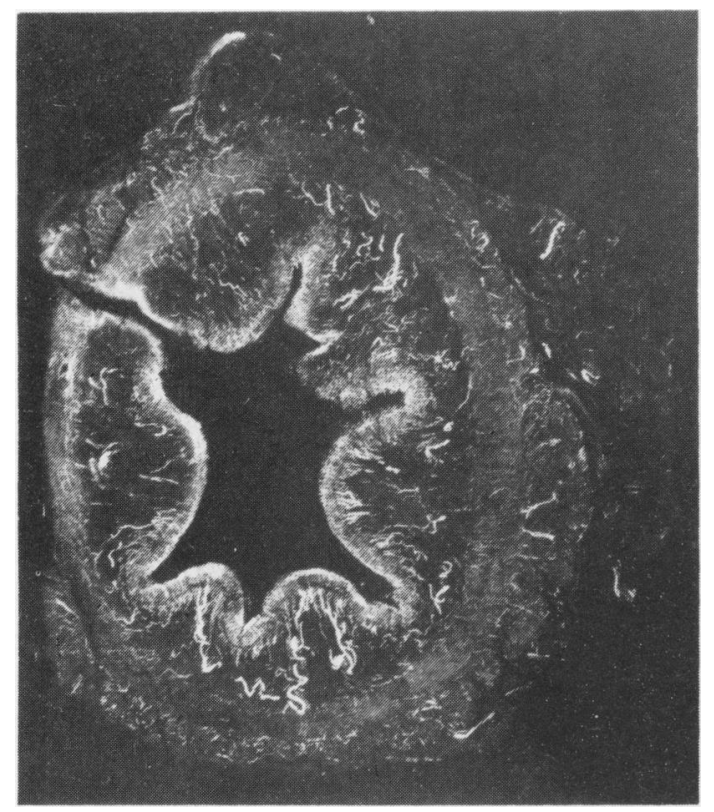

Fig. 2 Microvascular pattern in radiation stricture of sigmoid colon. Reduced vascularity all bowel wall layers. (Orig. mag. $\times 5$ )
In sections taken from peripheral areas of the specimen remote from the main lesion, there was straightening of the submucosal vessels and reduced vascularity most apparent in the submucosa. These changes were associated with submucosal oedema and fibrosis and non-occlusive intimal fibrosis in small arterial vessels in the bowel wall.

The mucosal vascular pattern was abnormal and the severity of change paralleled the reduction in vascularity in the remainder of the section. In the colon, localised areas of capillary ectasia situated adjacent to normal areas of mucosa were a common finding (Fig. 4). Other areas of the mucosa showed a loss of the normal regular capillary arrangement where mucosal regeneration had occurred. The first obvious change in small bowel mucosa was villous blunting and was seen in sections distant from the stricture or perforation. In more severely affected sections, abnormal villi were seen showing ectatic vessels and a reduction in the villous capillary network. In both the small and large bowel, there were areas of mucosa which were not perfused with contrast medium. Capillary microthrombi were present in these mucosal vessels with or without accompanying mucosal necrosis.

\section{FLUORESCENT RADIOGRAPH ANALYSIS}

Not less than five tissue samples from each of the 43 control and 22 radiation bowel damaged specimens were examined. The samples from the radiation bowel damaged specimens were all histologically abnormal but showed a variety of histological changes.

\section{Whole sample and layer vascularity}

The mean value obtained for barium concentration in whole tissue samples of the bowel wall from the 43 control specimens was $2.031 \pm 0.546 \%$. Barium concentration was $1.315 \pm 0.467 \%$ in the muscularis propria and $2.565 \pm 0.726 \%$ in the mucosasubmucosa. The radiation group showed a highly significant reduction in barium concentration in the whole tissue sample $(1.004 \pm 0.217 \% ; p<0.001)$, the muscularis propria $(0.677 \pm 0 \cdot 181 \% ; \mathrm{p}<0.001)$, and the mucosa-submucosa $(1.21 \pm 0.285 \% ; \mathrm{p}<0.001)$ when compared with the control group. The ratio between barium concentration in the mucosasubmucosa to that in the muscularis propria was $2.083 \pm 0.654$ in the control group and $1.905 \pm 0.573$ in the radiation group, but this difference was not statistically significant $(\mathrm{p}>0 \cdot 05)$.

A highly significant negative correlation between barium concentration and age was observed in the whole tissue samples from the 43 control patients $(\mathrm{r}=-0.669 ; \mathrm{p}<0.001)$. By contrast, no significant 

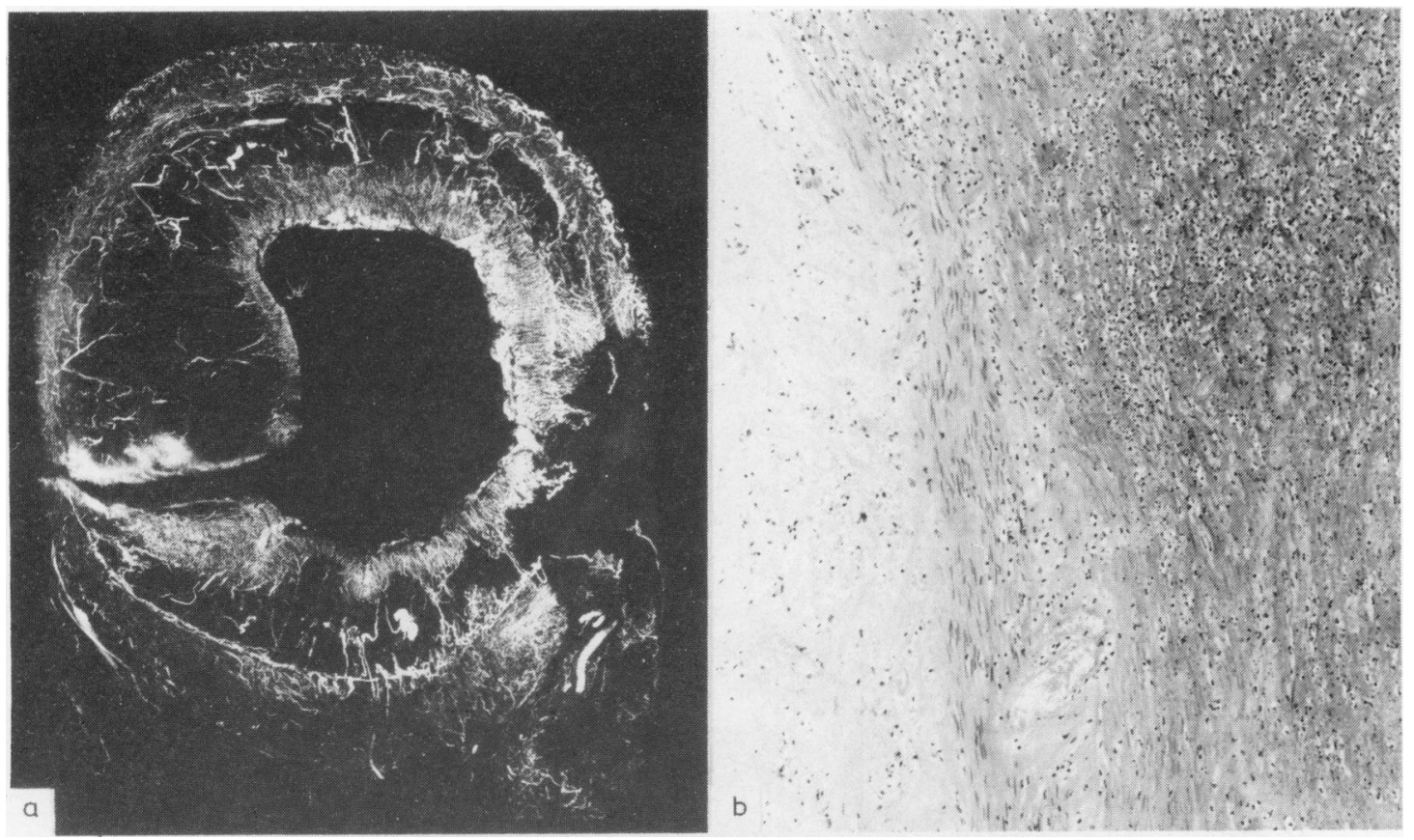

Fig. 3 (a) Microvascular pattern in radionecrosis of sigmoid colon. Avascular zone in bowel wall. (Orig. mag. $\times 5)$. (b) Photomicrograph of colonic wall at the periphery of the avascular zone shown in (a). (Orig. mag. $\times 100)$. Submucosa (left) and necrotic muscularis propria (right).

age correlation was observed in the radiation group for the whole sample (Fig. 5).

\section{Distance relationships}

(1) Terminal ileum: the relationship between barium concentration and the site in the specimen from which the whole samples were taken was investigated in four patients who had received combined intercavity and external radiotherapy and subsequently developed severe radionecrosis of the terminal ileum which necessitated ileal resection and right hemicolectomy. Five specimens of terminal ileum from the control group were also studied. The tissue samples were taken at measured distances from the ileocaecal valve (Fig. 6). No significant changes in barium concentration were observed in samples taken from different points in the normal terminal ileum and caecum. In radiation bowel disease, the maximum reduction in barium concentration was obtained in samples taken from within $15 \mathrm{~cm}$ of the ileocaecal valve where necrosis was present. Sample barium concentration remained below the $95 \%$ confidence limits of the control group for some distance proximally in ileum which appeared macroscopically less affected.

(2) Rectosigmoid colon: six specimens of radiation damaged rectosigmoid colon and five control specimens were studied in similar way. There was no significant change in barium concentration in samples obtained from different sites in the control specimens (Fig. 7). Stricture or necrosis was present in four specimens from patients who had received combined intracavity and external radiotherapy. In these specimens the lowest values for sample barium concentration were present 8-15 $\mathrm{cm}$ from the anorectal junction, but values outside two standard deviations of the control group were present in the more proximal sigmoid colon. By contrast a different pattern was observed in samples from the two patients who received intracavity radiotherapy alone. Barium concentration was reduced at the site of the main lesion but rapidly returned to normal in the proximal sigmoid colon.

\section{Discussion}

The incidence of necrosis of the intestine was much higher in this series of patient than previously documented cases of radiation bowel disease. Other authors have found a greater proportion of patients with stricture occurring at a similar time interval after pelvic radiotherapy. ${ }^{8}$ Histological examination 

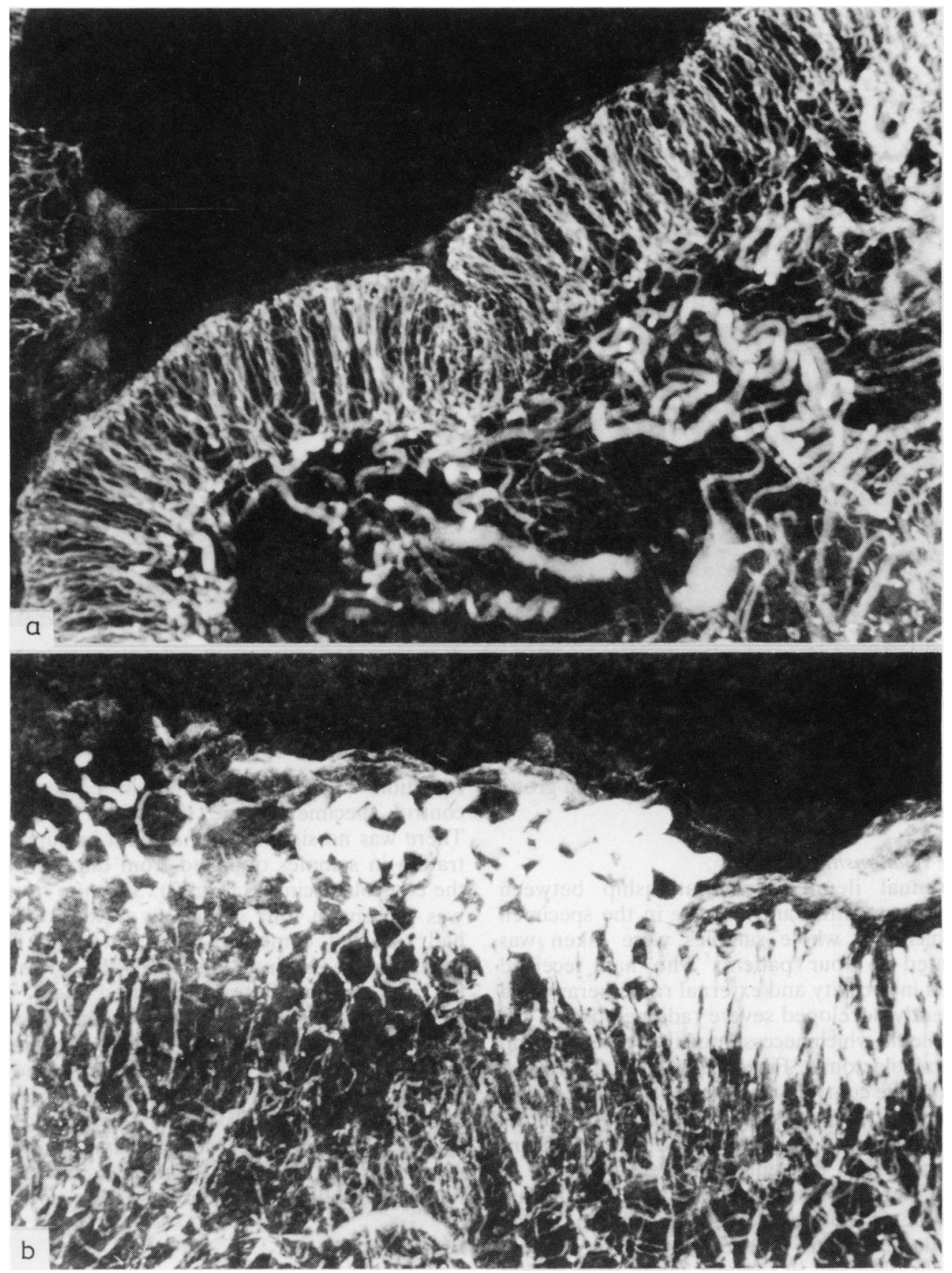

Fig. 4 Microradiographic appearances of normal colonic mucosa (top) and mucosa in radiation disease of the sigmoid colon (bottom). (Orig. mag. $\times 70$ ) 


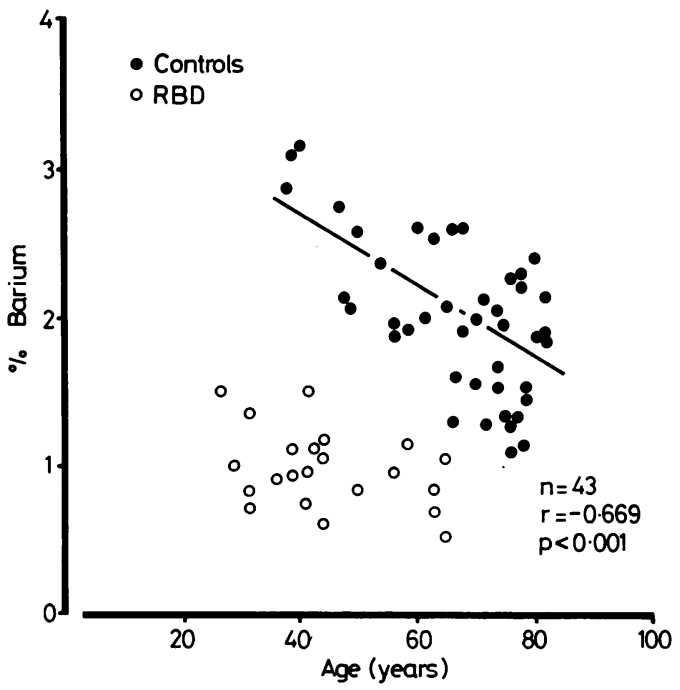

Fig. 5 Comparison between microvascular volume and age in the normal bowel $(n=43)$ and radiation bowel disease $(n=22)$.

of bowel after resection for radiation bowel disease in the past has shown clear evidence of occlusive changes in the intramural blood vessels ${ }^{29}$ and these observations have been supported in the present study.

Histological examination alone gives a less clear view of the vascularisation of the bowel wall than intravascular studies. Denker et al ${ }^{10}$ studied patients with bowel symptoms after pelvic irradiation by mesenteric angiography. They showed reduced

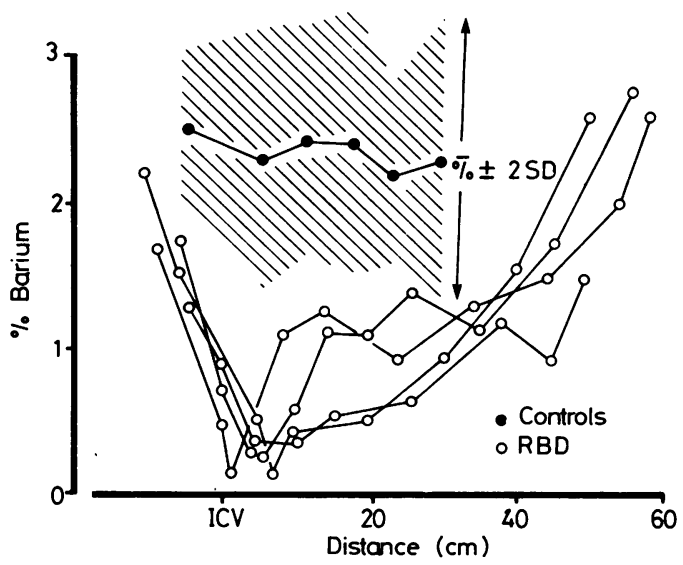

Fig. 6 Relationship between microvascular volume and linear distance in tissue sample from normal bowel and radionecrosis of terminal ileum.

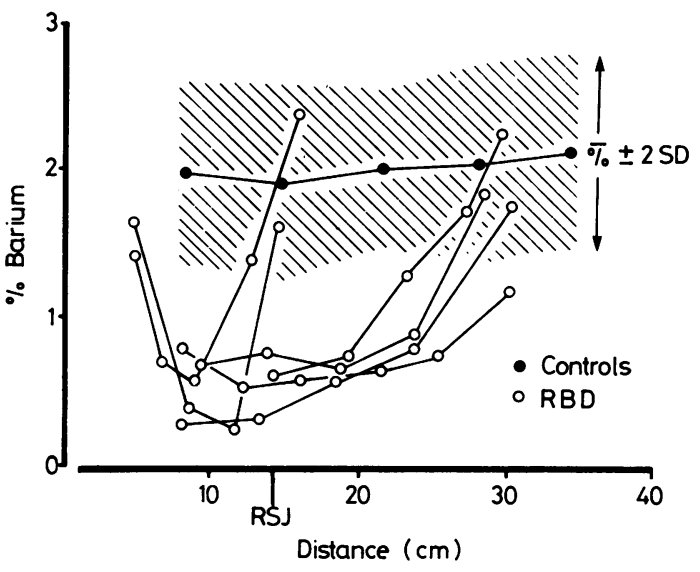

Fig. 7 Relationship between microvascular volume and linear distance in tissue samples from normal bowel and radiation disease of rectosigmoid colon.

vascularity of the bowel wall but did not show fine detail. Microradiography of excised specimens gives excellent visualisation of the intramural vasculature and while it has been used in animals, the method has been little used to study radiation bowel disease in man. The technique was originally used by Eddy and Casaret $\mathrm{t}^{5}$ to study the effects of small vessel disease on overall tissue vascularity in irradiated segments of rat small intestine. They showed vessel occlusions and a patchy reduction in vascularity of the bowel wall which occurred within the first week after exposure. Similar alterations in the microvasculature of the cat intestine were observed by Eriksson $^{6}$ in the early postirradiation period, but the subsequent appearances depended upon the initial dose of radiation administered. While complete recovery followed low doses, high doses caused reduced vascularity and alterations in the mucosal vascular pattern which persisted for four months.

The later effects of irradiation on the intestinal microvasculature in the human bowel have received less attention although Denker et al ${ }^{10}$ in their angiographic study of human radiation bowel disease showed reduced vascularity many months or years after therapeutic irradiation. The findings from the present microradiographic study support this observation and indicate that alterations in mucosal vascular architecture are present in human radiation bowel disease. A finding from the present investigation which was not evident in any of these previous studies was the presence of completely avascular zones of the bowel wall corresponding to 
areas of infarction. This may explain the high incidence of perforation in our patients.

The use of a radiograph fluorescence system for the estimation of microvascular volume in tissue samples ${ }^{7}$ was advantageous in providing information which could be analysed statistically to confirm the subjective impression of reduced vascularity at the site of radiation bowel injury, and to show that this reduction is similar in both bowel wall layers. This evidence lends support to the hypothesis that the occlusive lesions of the small intramural vessels commonly found in human radiation bowel disease produce a profound reduction in microvascular space leading to progressive ischaemia.

The sites of most severe injury after pelvic irradiation are in the lower sigmoid colon and upper rectum, probably because of their proximity to the field of radiation. " Overt injury to the small intestine is less common, but when it does occur, it is usually situated $6-10 \mathrm{~cm}$ from the ileocaecal valve. ${ }^{12}$ In the present study, these areas showed the most severe derangement of vascular architecture and maximum reduction in microvascular volume.

De Cosse et $\mathrm{l}^{4}$ stressed that the degree of involvement from radiation damage is far more extensive than is apparent on gross inspection. The majority of our patients received combined intracavity and external radiotherapy and a reduction in vascularity was present for some distance proximal to the main lesion, in segments of bowel which did not appear grossly abnormal. These findings have important therapeutic implications in that extensive resection may be necessary in order to remove all the affected bowel and thereby prevent anastomotic dehiscence or progression of the disease. Johnson et $a l^{13}$ observed that radiation bowel damage is much more localised if it occurs after treatment by intracavity irradiation alone and there is some evidence to support this in the present study.

Although several theories concerning the aetiology and pathogenesis of the delayed effect of radiation on the intestine have been postulated, ${ }^{14} 15$ the findings from the present investigation support those who believe that intramural vascular disease and progressive ischaemia are important factors in the natural history of human radiation bowel disease.
The authors wish to thank Mr A Curry and Miss P Rowland for their invaluable help in the preparation of the illustrations in this paper, and Mr B Faragher for statistical analysis of the results.

\section{References}

1 Localio SA, Pachter HL, Gouge TH. The radiationinjured bowel. Surg Ann 1979; 11: 181-205.

2 Berthrong M. Fajardo C. Radiation injury in surgical pathology. Am J Surg Pathol 1981; 5: 153-78.

3 Ashbaugh DG, Owens JC. Intestinal complications following irradiation for gynaecological cancer. Arch Surg 1963; 87: 100-5.

4 De Cosse JJ, Rhodes RS, Wents WB et al. The natural history and management of radiation induced injury of the gastro-intestinal tract. Surg Ann 1969; 170: 369-84.

5 Eddy HA. Casarett HGW. Intestinal vascular changes in the acute radiation syndrome. In: Sullivan M, ed. Gastro-intestinal radiation injury. New York: Excerpta Medica Foundation, 1968: 385-95.

6 Eriksson B. Microangiographic pattern in the small intestine of the cat after irradiation. Scand J Gastroenterol 1982; 7: 887-95.

7 Carr ND, Schofield PF, Pullan BR. A simple method for the estimation of microvascular volume in tissue samples. J Clin Phys Physiol Meas 1984. (In press.)

8 Jackson BT. Bowel damage from radiation. Proc $R$ Soc Med 1976; 69: 683-6.

9 Perkins DF. Spjut HJ. Intestinal stenosis following radiation therapy. Am J Radiol 1962; 88: 953-66.

10 Denker $\mathrm{H}$, Holmdow $\mathrm{KH}$. Lunderquist A et al. Mesenteric angiography in patients with radiation injury of the bowel after pelvic radiation. Am J Radiol 1972; 114: 476-81.

11 Kott I, Luca I. Kesler H. Gastrointestinal complications after therapeutic irradiation. Dis Colon Rectum 1971; 14: 200-5.

12 Marston A. Focal ischaemia of the small intestine. In: Intestinal ischaemia. London: Arnold, 1977: 136.

13 Johnson JE. Bladder and intestinal injuries following radiation therapy of carcinoma of the uterine cervix. Acta Rad Ther Phys Biol 1976; 15: 541-5.

14 Novak JM. Collins JT. Donowitz M et al. Effects of radiation on human intestinal tract. $J$ Clin Gastroenterol 1979; 1: 9-16.

15 Schier J, Symmonds RF. Dahlin DC. Clinicopathological aspects of actinic enteritis. Surg Gynecol Obstet 1964; 119: 1019-22. 